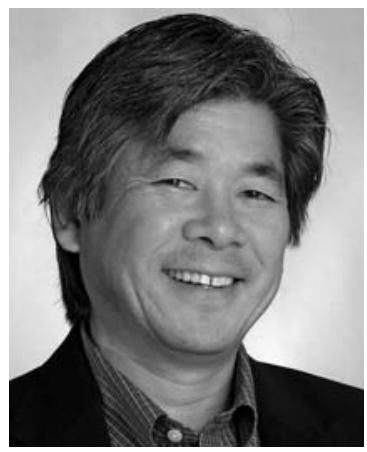

\title{
A Conversation with Richard Morimoto
}

\author{
INTERVIEWER: JIAYING TAN \\ Scientific Editor, Cell
}

Richard Morimoto is the Bill and Gayle Cook Professor of Biology in the Department of Molecular Biosciences and Director of the Rice Institute for Biomedical Research at Northwestern University.

Jiaying Tan: You're a pioneer in the discovery of heat shock proteins and set the stage for understanding the stress response. What got you interested in this?

Dr. Morimoto: When I was a graduate student at the University of Chicago I heard Matt Meselson, who was one of the discoverers of the heat shock response. At that point, they had observed a set of newly synthesized proteins on a gel that corresponded to the chromosome puffs when you heat shock Drosophila. This was just before any genes had been cloned and I wondered, "What does a molecular thermometer look like? Why should a fruit fly have a thermometer and what is it used for?" I'm continuing to ask these questions, but now I am back studying this in a living organism to understand not just how cells detect stress signals but also how these stress responses are integrated across tissues.

Jiaying Tan: How is the field moving in terms of the big questions?

Dr. Morimoto: Heat shock genes were among the first eukaryotic genes cloned in molecular biology, but there were still a lot of unanswered questions. What is the heat shock response? How are these heat shock genes activated? And, of course, what is the function of heat shock proteins? We had observations to suggest that heat shock genes and the heat shock response were tied to various chronic conditions including diabetes, heart disease, cancer, neurodegeneration, aging. However, we didn't have enough information to understand how or why, but people were starting to observe that heat shock genes could be turned on or off in unexpected conditions in response to diverse forms of acute and chronic stress conditions. As that field grew, the concept of molecular chaperones exploded: the idea that proteins need chaperones to fold properly. That was the "aha!" moment. Now it makes sense because we know that chaperones and quality control of protein homeostasis is a critical marker of health. I think whereas before people were focused only on transcriptional regulation, the big questions now involve proteostasis and how the network of cellular processes regulated by proteostasis determines the health of proteins.

Jiaying Tan: Can you define the causes and consequences of neurodegenerative and age-related disease in terms of proteostasis?

Dr. Morimoto: Aging leads to a general decline in quality control and the heat shock response, which leads to an imbalance in proteostasis. For example, in aging metazoans - and I think this is also true in yeast - when you see a decline in quality control systems, you see a reduction in chaperones and stress responses. This leads to an accumulation of misfolded and aggregated proteins. It is reasonable to assume that's probably not good for the health of the cell and that this would contribute to the risk for Alzheimer's, Parkinson's, amyotrophic lateral sclerosis, and hundreds of other diseases affecting other tissues. The question then becomes how much change the system will tolerate, and for how long. I posit that understanding age-related declines tells us by how much we need to tweak the system. It could be that you just need to increase folding or the proteosome or autophagy by $5 \%-15 \%$. Too often, there is a tendency to wait until the system collapses entirely and then we want to bring the whole boat back up. A boat that's sunk completely can be very hard to raise, but if you know where the leaks are and you repair it, the boat might stay afloat longer.

Jiaying Tan: Can this be used to find diagnostic markers for diseases?

Dr. Morimoto: I'm hopeful that we can identify the key components - the chaperones, the proteasome transport, and autophagy mechanisms - of the proteostasis network that go awry. Right now, there are too many genes, about 2000 that comprise the proteostasis network. We're trying to identify which of these are the critical nodes for quality control of the system. It could be that this system evolved such that there are a limited number of critical nodes of the proteostasis network that talk to each other and those are the ones that we have to identify 
and monitor. Such studies might inform us when the system is in decline well before we see profound functional decline. Instead of waiting until somebody is 75 years old and has dementia you could look at, for example, epithelial cells, to see if there's a decline in quality control capability and track whether it declines from year to year, very much like we do with blood tests, well before there is functional decline. That might lead to an approach where, when therapeutics become available, we could know how much to stimulate the system and to assess correction of proteostasis networks. You'd have to do it with epithelial or mononuclear cells either intact or transdifferentiated because obviously doing it with neurons from real brain tissue wouldn't be possible.

Jiaying Tan: Are these processes cell type-specific? Are there differences between progenitor cells and differentiated cells?

Dr. Morimoto: There will be significant differences in tissue and lineage specificity as it relates to proteostasis. There's no question that the proteome in a secretory cell is different than that of a neuron or a muscle cell. As you coevolve a proteome that has myofilaments and has myosin-actin contractility for assembly and disassembly, the proteins required to keep that proteome stable are going to be very different from those for an epithelial cell. We're just beginning to explore this in Caenorhabditis elegans and our lab is shifting to looking at the human cells as well, using the available GTEx data to compare expression of the proteostasis network in the brain, heart, intestine, and other tissues. Such comparative studies may provide us an overall understanding of collapse. As the system changes, what are the balances? What's the threshold? What are the risk factors? It is a form of precision molecular medicine based on a combination of biochemistry, functional assays to assess proteome health, and expression studies. You want to be able to take cells from an individual and ask how robust is the quality control system. If we combine the expression information on proteostasis with its functional properties, it may be possible to generate an index of their cellular health.

Jiaying Tan: Are these based on different peoples' genetic backgrounds or do they evolve during the aging process?
Dr. Morimoto: We know that genes and environment have to work together and we're learning a lot about epigenetics and about the role of diet and exercise. I imagine that in different parts of the world, those combinations would come together to play a critical role. That could be an interesting way to discover differences. My grandparents came to California from Japan in 1896. My DNA is still Japanese DNA but the environment in which I was raised is completely different. It will be interesting to understand those interactions.

Jiaying Tan: Do we know enough about proteostasis yet to apply that knowledge therapeutically?

Dr. Morimoto: We're getting closer. This field collectively has developed a deeper understading of the underlying molecular mechanisms. We have identified how molecular chaperones interact to regulate the folding and activity of different client proteins. For example, a number of pharmaceutical companies are working on Hsp90 inhibitors because if you block Hsp90 you clear these clients that are important for the rapid growth of cells; therefore, it's an anticancer therapy. They did not design it specifically as a proteostasis-based therapy, but this has become a wonderful example. Also, and we never expected this, you can inhibit Hsp90 - one of the most abundant chaperones that regulates hundreds of clients - and it doesn't kill the organism. That means that there's a concentration of Hsp90 inhibitor that actually works to target cancer cells. That gives us hope that you can reset the system. I think this is going to be a remarkable era where we can look at ageassociated degeneration, maybe track the progression of markers over time and shift the system back to a healthy state if there's a decline. The key for me is to understand the system and how it is regulated so that we do not change it abruptly. Rather the approach should be to retune the system so that instead of something declining in function at the age of 60 or 70 , it doesn't shift until 80 or 90 , in which case you've done your job.

I think quality control is something we all understand. We buy cars, we buy our computers, we buy almost all our technology with an eye toward quality control. You want something that is really well-made. The only things we don't control for quality are our own bodies. If we could increase healthy proteostasis, I think that will be good for us. 


\section{$\$_{\text {CSH\& }}^{\infty}$ Cold Spring Harbor Symposia SYMPOSIA On Quantitative Biology}

\section{A Conversation with Richard Morimoto}

Cold Spring Harb Symp Quant Biol 2015 80: 336-337

Access the most recent version at doi:10.1101/sqb.2015.80.030031

\section{License}

Email Alerting Receive free email alerts when new articles cite this article - sign up in Service the box at the top right corner of the article or click here. 